\title{
Gestión organizacional de atención a la diversidad y convivencia escolar en instituciones educativas públicas
}

\author{
Organizational management of attention to diversity and school coexistence in public educational institutions \\ Gestão organizativa da atenção à diversidade e à coexistência escolar nas instituições públicas de ensino
}

\section{RESUMEN}

La investigación tuvo como objetivo determinar la relación entre la gestión organizacional de atención a la diversidad y la convivencia escolar en las instituciones educativas públicas de nivel de secundaria de la Selva Central. El estudio es no experimental, de nivel descriptiva; se empleó el método científico e hipotético-deductivo, con un diseño correlacional comparativo. La muestra se constituyó a parir de 480 colaboradores, de las UGELs Chanchamayo, Pichanaki, Satipo, Pangoa, Río Tambo y Río Ene Mantaro; a quienes se aplicó dos cuestionarios, que fueron sometidos a validación y confiabilidad. Los hallazgos del análisis comparativo revelan que la mayoría de encuestados por UGEL y tamaño de institución educativa perciben un nivel bueno de gestión organizacional de atención a la diversidad y un nivel favorable de convivencia escolar. Como conclusión se destaca una relación directa, fuerte $(0,793)$ y altamente significativa $(* *, p<0,01)$ entre la gestión organizacional de atención a la diversidad y convivencia escolar.

Palabras clave: Gestión organizacional; Atención a la diversidad; Convivencia escolar; Instituciones educativas públicas; Colaboradores
ABSTRACT

The research aims to determine the relationship between the organizational management of attention to diversity and school coexistence in public educational institutions of secondary level of the Central Forest. The study is non-experimental, descriptive level; The scientific and hypothetico-deductive method was used, with a comparative correlational design. The sample was achieved by 480 collaborators, from the UGELs Chanchamayo, Pichanaki, Satipo, Pangoa, Río Tambo and Río Ene Mantaro; to whom two questionnaires were applied, which were subjected to validation and reliability. The results of the comparative analysis reveal that the majority of those surveyed by UGEL and the size of the educational institution, perceive a good level of organizational management of attention to diversity and a favorable level of school coexistence. In conclusion, a direct, strong (0.793) and highly significant $(* *, p<0.01)$ relationship stands out between the organizational management of attention to diversity and school coexistence.

Key words: Organizational management; Attention to diversity; School coexistence; Public educational institutions; Collaborators

\section{RESUMO}

A pesquisa visa verificar a relação entre a gestão organizacional da atenção à diversidade e a convivência escolar em instituições públicas de ensino de nível médio da Floresta Central. O estudo é não experimental, nível descritivo; foi utilizado o método científico hipotéticodedutivo, com desenho correlacional comparativo. A amostra foi realizada por 480 colaboradores, das UGELs Chanchamayo, Pichanaki, Satipo, Pangoa, Río Tambo e Río Ene Mantaro; a quem foram aplicados dois questionários, os quais foram submetidos à validação e confiabilidade. Os resultados da análise comparativa revelam que a maioria dos pesquisados pela UGEL e o porte da instituição de ensino percebem um bom nível de gestão organizacional de atenção à diversidade e um nível favorável de convivência escolar. Em conclusão, destaca-se uma relação direta, forte $(0,793)$ e altamente significativa ${ }^{* *}, \mathrm{p}$ $<0,01)$ entre a gestão organizacional da atenção à diversidade e a convivência escolar.

Palavras-chave: Gestão organizacional; Atenção à diversidade; Vida escolar; Instituições educacionais públicas; Colaboradores 


\section{INTRODUCCIÓN}

En el sistema educativo peruano, más aún en el ámbito regional y local, se hace latente el escaso reconocimiento de la sociedad como un grupo social y culturalmente diferenciada, por lo que, considerando el devenir histórico, urge la necesidad de poner las bases para la construcción de un proyecto educativo de la región Junín, que atienda a las demandas y necesidades educativas de la diversidad cultural, étnica, lingüística, de género, de necesidades educativas especiales y situación migratoria. En este contexto, las instituciones educativas asentadas en la Selva Central vienen asumiendo el gran desafío en la atención de estudiantes de diferentes colectivos, con características y necesidades diversas, y para lograr la cohesión socioeducativa, tienen que brindar la atención equitativa e inclusiva a toda la población escolar, considerando su heterogeneidad y diversidad.

Así pues, realizada la revisión bibliográfica pertinente, es plausible sostener que la producción científica acerca del tema de la diversidad y su correlato con la convivencia escolar, en el tópico de su prevención, aún no responde con soltura a las necesidades y complejidades que se derivan de esta problemática asentada en el interior de las instituciones educativas del medio local y regional, menos de aquellas que se ubican en la selva central del país. En ese sentido, estudiar y analizar profunda y sistemáticamente factores como la diversidad y la convivencia escolar representa en la actualidad un importante desafío, tanto a nivel teórico como metodológico. Pues, el estudio se justifica en la necesidad de comprender amplia y profundamente la relación entre la atención a la diversidad y la convivencia escolar en las instituciones educativas para garantizar una auténtica formación integral de las y los estudiantes de la región Junín.
En ese contexto, el objetivo del estudio fue determinar la relación entre la gestión organización de atención a la diversidad y la convivencia escolar en las instituciones educativas públicas de nivel secundaria de la Selva Central, y comparar las diferencias en el nivel de la gestión organizacional de atención a la diversidad y el nivel de convivencia escolar en las instituciones educativas públicas de nivel secundaria de la Selva Central por tamaño de la institución educativa y UGEL.

La revisión de los estudios previos realizados en diferentes contextos, aportan a la comprensión de las investigaciones y avances en torno a la gestión organizacional de atención a la diversidad y convivencia escolar en las instituciones educativas. En esta perspectiva, autores como Vargas (2019) en su tesis doctoral, estudió la gestión de la diversidad y la calidad del servicio educativo en los colegios de la provincia de Lucanas, región Ayacucho, en una muestra constituida por 37 profesores y 370 estudiantes del nivel secundaria. El estudio corresponde a un enfoque cuantitativo, de nivel descriptivo correlacional. Los resultados demostraron una correlación altamente significativa entre la gestión de la diversidad y la calidad del servicio educativo, lo que implica a mejor gestión de la diversidad, mayores son las condiciones básicas de la infraestructura, la seguridad, y la enseñanza y aprendizaje.

Por su lado, Arias (2018) en Colombia, realizó un trabajo denominado: "Ambientes escolares: un espacio para el reconocimiento y respeto por la diversidad". El estudio fue aplicado en 33 estudiantes de grado once de una institución pública de la ciudad de Armenia-Quindío; el propósito fue proponer ambientes escolares de comunicación e interacción; mediante la estrategia metodológica de la investigación acción participativa, que posibilitarían identificar la diversidad y restablecer 
la convivencia escolar. La investigación refleja las realidades de los alumnos dentro y fuera del centro escolar, así como los desafíos que afrontan los profesores, para generar ambientes escolares adecuados en la institución educativa desde y para la otredad. El aporte refleja un examen de la significancia de los ambientes escolares planificados, que restablecen relaciones favorables que fortalecen la convivencia escolar.

Jiménez y Montecinos (2018) en Chile, realizó una investigación titulada: "Diversidad, modelos de gestión y formación inicial docente: desafíos formativos desde una perspectiva de justicia social". En este estudio examinaron enfoques donde docentes inexpertos gestionan la diversidad escolar y la articulación entre estos, y su formación pedagógica inicial. Los resultados evidencian dos enfoques de gestión de la diversidad: el primero en torno a las necesidades educativas especiales del estudiantado y el segundo orientado al refuerzo de las relaciones humanas; además revelaron insuficiente formación inicial en competencias pedagógicas para laborar en contextos educativos diversos. Finalmente, los resultados revelan que dichos enfoques responden a modalidades reduccionistas de gestión, debido a la escasa articulación interdisciplinar en la formación docente.

Begué (2018) en su tesis doctoral: "La atención a la diversidad en los centros educativos de la comunidad de Madrid. Análisis de la relación entre el texto y el contexto", realizó un estudio descriptivo en 532 docentes y 187 madres y padres de la comunidad de Madrid. El estudio ha encontrado limitaciones en los docentes para poder llevarla a la práctica la inclusión de los estudiantes en las aulas; no obstante, sus percepciones, creencias y actitudes actúan como facilitadores de la implementación de prácticas inclusivas en los centros educativos. Contrariamente en la apreciación de los padres de familia, dichos profesores muestran actitudes negativas frente a la diversidad, lo que dificultan la inserción de sus hijos en la escuela ordinaria. Concluye que los profesores perciben a la escuela inclusiva como una organización para atender a las necesidades y características de la diversidad en colaboración de la comunidad educativa. En esta línea, los maestros asumen actitudes positivas frente a la diversidad del estudiantado.

En España Luzón et al. (2009) han publicado el artículo titulado: "Buenas prácticas en los programas extraordinarios de atención a la diversidad en los centros de educación secundaria. Una mirada desde la experiencia”. Los hallazgos del estudio reflejan que los profesores desarrollan "buenas prácticas pedagógicas" dar respuesta a los riesgos de exclusión escolar en las instituciones de nivel secundaria; esto implica la presencia del equipo docente altamente motivado y comprometido, con espíritu innovador y profesionalismo para atender la diversidad; así como el equipo directivo con liderazgo educativo y formación pedagógica que impulsan la marcha de proyectos integrados que favorecen ciertamente una mayor presencia del estudiantado con riesgo de exclusión.

Aragón (2019) presentó la investigación sobre la "Convivencia escolar: Una mirada desde la educación social" con el objetivo de analizar y describir los factores que alteran la convivencia escolar en las instituciones escolares y estrategias de resolución para la mejora. Los principales hallazgos del estudio dan cuenta que los profesores no están capacitados para abordar los problemas de convivencia que se manifiestan en el contexto escolar; no obstante, considera propicio la práctica del deporte, la música y el baile fuera del ámbito escolar para fortalecer una convivencia favorable y desarrollar valores utilitarios. Concluye considerando favorable la complementariedad de 
la educación social que se dan fuera de los centros educativos, a aquellos que imparten los profesores en el ámbito escolar.

Bustamante (2019) desarrolló la investigación titulada: "Gestión Escolar y convivencia escolar en el colegio Cesar A. Vallejo - La Victoria”. La metodología utiliza fue descriptivo correlacional y diseño no experimental, constituida por una muestra de 108 docentes de educación básica. Los resultados hallados demostraron que existe relación significativa entre la gestión educativa y la convivencia escolar en la población estudiada; además se corroboran que el $30 \%$ de encuestados manifiestan la convivencia escolar es de nivel buena a consecuencia del nivel moderadamente eficiente que se percibe a la gestión educativa en el colegio Cesar A. Vallejo del distrito de la Victoria -UGEL 03 Lima.

Moncada-Herrera (2018) investigó la "gestión de convivencia escolar: del aseguramiento a la necesidad de fortalecer la formación". El objetivo fue examinar las prácticas de gestión de la convivencia en establecimientos escolares. El estudio es de corte cuantitativo y diseño no experimental, utilizó un cuestionario de Convivencia Escolar para ser observado en 1408 alumnos de educación básica. Luego de un análisis comparativo encontró para todas las dimensiones diferencias significativas, según el tipo de institución; la "formación para la no violencia" resultaron las más debilitadas, mientras las prácticas formales de "aseguramiento" son las más fortalecidas. Los resultados concluyen en la necesidad de fortalecer la formación en todas las instituciones para fomentar ambientes escolares donde todos aprendan y se sientan bien tratados.

En Perú; Quispe, Robinet y López (2018) estudiaron cualitativamente los significados $\mathrm{y}$ prácticas de convivencia democrática e inclusiva en una muestra poblacional de 5 docentes, 2 directivos y 8 estudiantes que cursan el 4to de secundaria en la IE pública de Ayacucho. En los resultados se destacan la aplicación de las normas, la participación colectiva, los valores, las decisiones consensuadas, la inclusión y el respeto a la diversidad en la práctica de la convivencia escolar; asimismo la interacción en las actividades recreativas y el trato horizontal entre estudiantes y profesores propician una convivencia democrática e inclusiva favorable al interior de la institución educativa; no obstante, el maestro ejerce su poder hacia estudiante. Las iniciativas pedagógicas que promueven las relaciones interpersonales lineales y el compromiso de los actores educativos constituyen estrategias favorables para la mediación y solución de los conflictos.

Por otra parte, Ramírez (2017) en el Perú, presentó su trabajo de investigación con el objetivo de establecer "la relación entre la gestión institucional y la convivencia escolar en la institución educativa Alberto Leveaú García” de la provincia de Picota". Los hallazgos en $51 \%$ de los encuestados manifiestan una gestión deficiente, en tanto el $66 \%$ de la población estudiada consideran poco favorable la convivencia escolar; estos resultados se explican a menor información y compromiso que tiene la comunidad educativa respecto a la visión institucional y los objetivos estratégicos; mayor es el riesgo de ocasionar una convivencia escolar desfavorable. Por otro lado, el estudio concluye "la gestión institucional se relaciona positiva y significativamente con la convivencia escolar", el cual implica que los mecanismos de comunicación interna, las alianzas interinstitucionales, el gobierno escolar inherente a la gestión institucional genera directamente una convivencia escolar favorable.

Retuert y Castro (2017) en Chile presentaron un artículo con el propósito de "interpretar las teorías subjetivas que preparan los profesores de enseñanza 
media de Liceos de la ciudad de La Serena, respecto a su rol profesional en la construcción de convivencia escolar". El estudio fue de corte cualitativo y enfoque interpretativo, que utilizó la entrevista grupal para recopilar los datos en una muestra poblacional de 30 docentes de cuatro centros escolares. Los hallazgos encontrados evidencian que los profesores consideran a sus estudiantes con diversas necesidades, lo que implicaron un cambio de concepción y adopción del rol docente para establecer las dinámicas de la convivencia escolar; a través de la retroalimentación y validación constante con la experiencia laboral y profesional. Enfatizando en el desarrollo de la formación inicial $y$ en servicio docente para nutrir las competencias didácticas que ayude a regular la disciplina en el aula y la reconstruir la convivencia escolar.

Los Objetivos de Desarrollo Sostenible (ODS) planteados por las Organización de las Naciones Unidas con metas al 2030, en particular el ODS 4, exhorta a los países "Garantizar una educación inclusiva, equitativa y de calidad y promover oportunidades de aprendizaje durante toda la vida para todos" (ONU, 2017, p. 38). Cabe destacar que, el Perú como miembro activo de la Asamblea General de las Naciones Unidas se alinea en este Acuerdo Nacional. El objetivo de la política de Estado 11, propone la igualdad de oportunidades de educación para las mujeres, los discapacitados, estudiantes de comunidades étnicas y otros; en este mismo sentido, la Meta 4.5 plantea asegurar para el 2030 el acceso a la educación de las personas vulnerables en igualdad de condiciones, incluidos los estudiantes con discapacidad y procedentes de pueblos indígenas (ONU, 2017) brindándoles entornos de aprendizaje seguros e inclusivos, con instalaciones educativas que respondan a las características y necesidades de estudiantes con discapacidad.
La Ley General de Educación №28044 adopta la inclusión educativa como uno de sus principios, que orienta hacia la incorporación de "las personas con discapacidad, grupos sociales excluidos, marginados y vulnerables, especialmente en el ámbito rural, sin distinción de etnia, religión, sexo u otra causa de discriminación, contribuyendo así a la eliminación de la pobreza, la exclusión y las desigualdades" (Congreso de la República, 2003, p. 2). Asimismo, instituye programas especiales que aseguran la igualdad de oportunidades y equidad de género en el ámbito rural.

Si bien, las políticas educativas planteadas en el Proyecto Educativo Nacional al 2036 aseguran la igualdad de oportunidades de aprendizaje y desarrollode todoslasylosestudiantes, reconociendo y valorando su diversidad, considerando sus características, necesidades e intereses de las diferencias, y sobre todo priorizando la atención de poblaciones en situación de vulnerabilidad asociada a su origen étnico, condición socioeconómica, género, población de ámbitos rurales, población migrante, población en estado de abandono físico y moral, personas con discapacidad y necesidades educativas especiales (Consejo Nacional de Educación, 2020), que aseguran el pleno ejercicio de los derechos individuales y colectivos en igualdad de condiciones.

En este marco de análisis, la atención a la diversidad se muestra como la piedra angular que invita a reflexionar sobre sus praxis y reales alcances en el escenario educacional (Cepeda, 2018). Por consiguiente, es de imperiosa necesidad reorientar la práctica educativa con el propósito de dar respuesta a la población escolar tan diversa y heterogénea. En tanto, la educación siendo un derecho para todos, es imprescindible que las respuestas educativas se ajusten a las necesidades de todos las y los estudiantes (Cornejo, 2017), respetando las 
diferencias culturales, étnicas, lingüísticas, sociales y capacidades cognitivas y físicas; que garantizan la igualdad de oportunidades a todos y todas, ante el derecho superior a la educación; además de las adecuaciones o adaptaciones curriculares y apoyos educativos que se brindan.

De acuerdo a lo anterior, Magro-Gutiérrez (2019) refiere que los docentes requieren adquirir durante su formación las competencias pedagógicas para educar a la diversidad y grupos heterogéneos, desarrollando adaptaciones pedagógicas, adecuaciones curriculares, gestión del tiempo, espacios y recursos didácticos y evaluación formativa. En realidad, se requiere de la formación magisterial en el marco de un enfoque de justicia social, para brindar atención educativa a la diversidad; bajo un principio de respeto a la diferencia y una oportunidad de aprendizaje colectivo (Álvarez, 2018; Maldonado, 2018). Bajo esta perspectiva, el rol del docente y su formación inicial y en servicio, cobra una singular relevancia en las prácticas pedagógicas para dar respuesta a la diversidad en el aspecto organizativo, metodológico y curricular (Habib y Hernández, 2020).

Si bien es cierto, la diversidad está constituida por la multiplicidad de características que hacen diferente a las personas y grupos en relación a factores biológicos, genéticos, culturales y sociales (Arnaiz, 2003; Fregoso, 2019). En esta línea, varias investigaciones han coincidido en comprender la diversidad como las diferencias individuales y personales que distinguen a cada estudiante de los demás, como ser único, singular, irrepetible y múltiple a la vez, por su propia característica que se generan de su constitución biológica y cultural (Morin, 2000; Cornejo, 2017; Hernández-Prados e Ibáñez-Bordallo, 2018; Castro-Rodríguez y EirínNemiña, 2018; Cornejo-Valderrama et al. 2017).
La convivencia escolar viene formando parte de recientes políticas educativas a nivel del mundo y Latinoamérica; entre los acuerdos internacionales implementados para promover la convivencia escolar se encuentra la declaración de la Organización de Naciones Unidas (ONU) para la promoción de una cultura para la paz y la no violencia para los niños del mundo 2001-2010 (ONU, 1999) y la Declaración Universal de los Derechos del Niño (ONU, 1959). Sobre la base de estos planteamientos, los principios de la educación peruana inspiran el pleno respeto a las normas de convivencia, que fortalecen la conciencia moral individual y una sociedad basada en el ejercicio constante de la responsabilidad ciudadana; así como el reconocimiento y respeto a las diferencias para la convivencia armónica (Congreso de la República, 2003).

La mejora de la convivencia escolar se logra transformando las prácticas escolares y las políticas de gestión en la escuela. Desde esta postura la convivencia no puede explicarse sólo a través de las relaciones interpersonales que se dan en dicho contexto, sino que es necesario observar la organización institucional; es decir, las formas en que interactúan los actores educativos guiadas por las políticas y prácticas educativas establecidas institucionalmente (Ochoa y Díez-Martínez, 2012; citado en Ochoa y Pérez, 2019).

En ese sentido, las buenas prácticas para la mejora permanente de las relaciones armónicas y la convivencia democrática se pueden impulsar; a través de actividades y espacios: asambleas de aula, paseo campestre, festidanzas, recreos amigables, actividades deportivas, entre otros que la institución educativa estime convenientes (Minedu, 2020). Así también, la formación en habilidades sociales y emocionales, la educación en valores, la 
elaboración conjunta de normas, una metodología que facilite la cohesión de grupo y la figura del profesor como referente moral; favorecen en la mejora de la convivencia pacífica y democrática; además, de la mediación y el dialogo y como vía de solución de conflictos. De ahí la importancia, en las instituciones educativas de enseñar a las y los estudiantes a convivir y dotarlos de habilidades blandas para asegurar la formación en toda su plenitud y potencialidad (Rojas, 2020).

\section{MÉTODO}

El estudio corresponde a una investigación no experimental, de nivel descriptivo, con un diseño correlacional comparativa; debido a que se observan las correlaciones y se comparan los niveles organizacionales de atención a la diversidad y convivencia escolar, por tamaño de institución educativa y UGEL. Para el desarrollo de la investigación se utilizaron el método científico, que valiéndose de sus procedimientos, buscó explicar el problema; a través de la observación, la verificación y el análisis objetiva de los datos, y el planteamiento de las conclusiones generales; además se utilizó el método hipotético-deductivo que guio en el planteamiento de las hipótesis y en adelante la contrastación de las mismas y el método correlacional que permitió analizar las variables del estudio; a través del coeficiente correlación; en tanto esta correlación entre las variables es de tipo asociativa.

En el presente estudio la muestra fue constituida por 480 trabajadores distribuidos entre directivos y docentes de las instituciones educativas públicas urbanas de nivel secundaria de la Selva Central, que pertenecen a las Unidades de Gestión educativa Local de Chanchamayo, Pichanaki, Satipo, San Martin de Pangoa, Rio Ene Mantaro y Rio Tambo. Para el estudio de las variables y el proceso de recolección de la información se utilizó la técnica de encuesta virtual, y para la indagación de las percepciones de la gestión organizacional de atención a la diversidad y la convivencia escolar, se aplicaron a los directivos y docentes dos instrumentos que consistió en dos cuestionarios virtuales que contienen preguntas con opciones de respuestas, las cuales representan a las variables de estudio y que sirvieron para la medición correspondiente: cuestionario de las percepciones sobre la atención a la diversidad y cuestionario para evaluar la percepción de la convivencia escolar; que fueron validados con el coeficiente de concordancia $\mathrm{V}$ de Aiken para la opinión de los expertos, mientras que la validez de constructo con el coeficiente de correlación r de Pearson corregida, y la confiabilidad de los instrumentos se evalúo con los resultados de su aplicación a una muestra piloto y el coeficiente de consistencia interna Alfa de Cronbach.

\section{RESULTADOS Y DISCUSIÓN}

En este apartado se presentan los resultados del procesamiento estadístico realizado en torno a las correlacionales entre las variables: Gestión organizacional de atención a la diversidad y la convivencia escolar en los colaboradores de la muestra total de estudio $(\mathrm{n}=480)$.

\section{Análisis de la relación entre gestión organizacional de atención a la diversidad y convivencia escolar}

La relación entre gestión organizacional de atención a la diversidad y convivencia escolar, medida con el coeficiente de correlación por rangos rho de Spearman de 0,793 , indica que la relación entre estas variables es directa o positiva, fuerte $(0,7$ a 0,99$)$ y altamente significativa $(* *, p<0,01)$, lo que significa que, a mejor gestión organizacional de atención a la diversidad, mejor es la convivencia escolar, a menor gestión organizacional, menor convivencia escolar (Ver Tabla 1). 
Tabla 1. Coeficientes de correlación rho de Spearman entre gestión organizacional de atención a la diversidad y convivencia escolar $(n=480)$.

Convivencia escolar

Gestión organizacional

$0,783^{* *}$

$\left({ }^{\star *}\right)$ : Altamente significativo $(\mathrm{p}<0.01)$.

Análisis de la relación entre gestión convivencia escolar es directa, fuerte y altamente organizacional de atención a la diversidad significativa, entre 0,737 en Satipo y 0,832 en Río y convivencia escolar por variables de Tambo (Tabla 2).

\section{comparación}

Por UGEL, la relación entre la gestión organizacional de atención a la diversidad y la

Tabla 2. Coeficientes de correlación rho de Spearman entre gestión organizacional de atención a la diversidad y convivencia escolar, por UGEL $(n=480)$.

\begin{tabular}{lc}
\hline \multicolumn{1}{c}{ Gestión organizacional } & Convivencia escolar \\
\hline Chanchamayo (290) & $0,801^{\star \star}$ \\
Pangoa (69) & $0,800^{\star *}$ \\
Pichanaki (10) & $0,794^{\star \star}$ \\
Río Ene Mantaro (11) & $0,741^{\star \star}$ \\
Río Tambo (24) & $0,832^{\star \star}$ \\
Satipo (76) & $0,737^{\star \star}$ \\
\hline
\end{tabular}

$(* *)$ : Altamente significativo $(\mathrm{p}<0.01)$.

Por tamaño de institución educativa, la relación entre la gestión organizacional de atención a la diversidad y la convivencia escolar es directa, fuerte $y$ altamente significativa, entre 0,742 en instituciones medianas y 0,808 en instituciones pequeñas (Tabla 3).

Tabla 3. Coeficientes de correlación rho de Spearman entre gestión organizacional de atención a la diversidad y convivencia escolar, por tamaño de la I.E. $(n=480)$.

\begin{tabular}{ll}
\hline Gestión organizacional & Convivencia escolar \\
\hline Grande $(112)$ & $0,795^{* *}$ \\
Mediana (221) & $0,742^{* *}$ \\
Pequeña $(147)$ & $0,808^{* *}$ \\
\hline
\end{tabular}

$(* *)$ : Altamente significativo $(\mathrm{p}<0.01)$. 
Análisis del nivel de la gestión organizacional de atención a la diversidad

El nivel de la gestión organizacional de atención a la diversidad percibido por los trabajadores es bueno (85\%), el 13,8\% la percibe como aceptable y el 1,2\% deficiente (Tabla 4 ).

Tabla 4. Nivel de la gestión organizacional de atención a la diversidad ( $\mathrm{n}=480)$.

\begin{tabular}{|c|c|c|}
\hline Nivel & Trabajadores & $\%$ \\
\hline Deficiente & 6 & 1,2 \\
\hline Aceptable & 66 & 13,8 \\
\hline Buena & 408 & 85,0 \\
\hline
\end{tabular}

\section{Análisis del nivel de la convivencia escolar}

El nivel de la convivencia escolar percibido por los trabajadores es favorable (94,4\%), el 5,2\% percibe que es poco favorable y el 0,4\% desfavorable (Tabla 5).

Tabla 5. Nivel de la convivencia escolar $(n=480)$.

\begin{tabular}{lcc}
\hline \multicolumn{1}{c}{ Nivel } & Trabajadores & $\%$ \\
\hline Desfavorable & 2 & 0,4 \\
Poco favorable & 25 & 5,2 \\
Favorable & 453 & 94,4 \\
\hline
\end{tabular}

Análisis del nivel de la gestión organizacional de atención a la diversidad por criterios de comparación

La gestión organizacional por UGEL es buena y ligeramente mayor en Pangoa (94,2\%) y Río Tambo (91,7\%), que en Chanchamayo (84,5\%), Pichanaki (80\%), Satipo (80,3\%) y Río Ene Mantaro $(63,6 \%)$ (Tabla 6).

El nivel aceptable de la gestión organizacional oscila entre 5,8\% (Pangoa) y 36,4\% (Río Ene
Mantaro). El nivel deficiente de la gestión organizacional no supera el 3\%, sin casos en las UGEL de Pangoa, Pichanaki, Río Ene Mantaro y Río Tambo.

de la convivencia escolar percibido por los trabajadores es favorable (94,4\%), el 5,2\% percibe que es poco favorable y el $0,4 \%$ desfavorable (Tabla 5). 
Tabla 6. Nivel de la gestión organizacional de atención a la diversidad por UGEL $(\mathrm{n}=480)$.

\begin{tabular}{lccc}
\hline \multicolumn{1}{c}{ UGEL / Nivel } & Nivel (\%) & Buena \\
\hline Chanchamayo (290) & Deficiente & Aceptable & 84,5 \\
Pangoa (69) & 1,4 & 14,1 & 94,2 \\
Pichanaki (10) & & 5,8 & 80,0 \\
Río Ene Mantaro (11) & 20,0 & 63,6 \\
Río Tambo (24) & & 36,4 & 91,7 \\
Satipo (76) & 2,6 & 8,3 & 80,3 \\
\hline
\end{tabular}

La gestión organizacional por tamaño de institución educativa es buena y ligeramente mayor en las instituciones medianas $(88,7 \%)$, que en las instituciones pequeñas $(85,7 \%)$ y grandes $(76,8 \%)$ (Tabla 7).

El nivel aceptable de la gestión organizacional oscila entre 10,9\% (instituciones medianas) y 19,6\% (instituciones grandes. El nivel deficiente de la gestión organizacional no supera el $4 \%$, sin casos en la respuesta organizacional de las instituciones medianas y pequeñas.

Tabla 7. Nivel de la gestión organizacional de atención a la diversidad por tamaño de I.E. $(n=480)$.

\begin{tabular}{lccc}
\hline \multicolumn{1}{c}{ Tamaño de I.E. / Nivel } & Nivel (\%) & Buena \\
\hline Grande (112) & Deficiente & 19,6 & 76,8 \\
Mediana (221) & 3,6 & 10,9 & 88,7 \\
Pequeña (147) & 0,5 & 13,6 & 85,7 \\
\hline
\end{tabular}

Análisis del nivel de la convivencia escolar por criterios de comparación

La convivencia escolar por UGEL es favorable superior al 90\%, entre 90,9\% (Río Ene Mantaro) y 100\% (Pichanaki y Río Tambo) (Tabla 8). El nivel poco favorable de la convivencia escolar no supera el 10\% sin casos en Pichanaki y Río Tambo. El nivel desfavorable de la convivencia escolar no presenta casos, salvo en la UGEL Chanchamayo, que no supera el $1 \%$. 
Tabla 8. Nivel de la convivencia escolar por UGEL $(n=480)$.

\begin{tabular}{lccc}
\hline \multicolumn{1}{c}{ UGEL / Nivel } & Nivel (\%) & \\
& Deficiente & Aceptable & Buena \\
\hline Chanchamayo (290) & 0,7 & 5,9 & 93,4 \\
Pangoa (69) & & 1,4 & 98,6 \\
Pichanaki (10) & & 100 \\
Río Ene Mantaro (11) & 9,1 & 90,9 \\
Río Tambo (24) & & 100 \\
Satipo (76) & 7,9 & 92,1 \\
\hline
\end{tabular}

La convivencia escolar por tamaño de la institución educativa es favorable en más del $85 \%$, desde $88,4 \%$ (instituciones grandes) hasta $96,6 \%$ (instituciones pequeñas) (Tabla 9). El nivel poco favorable de la convivencia escolar alcanza el 10,7\%, mientras que el nivel desfavorable no supera el $1 \%$, sin casos en las instituciones pequeñas.

Tabla 9. Nivel de la convivencia escolar por tamaño de I.E. $(n=480)$.

\begin{tabular}{lccc}
\hline \multicolumn{1}{c}{ Tamaño de I.E. / Nivel } & Nivel (\%) & Buena \\
\hline Grande (112) & Deficiente & Aceptable & 88,4 \\
Mediana (221) & 0,9 & 10,7 & 95,9 \\
Pequeña (147) & 0,5 & 3,6 & 96,6 \\
\hline
\end{tabular}

\section{Prueba de hipótesis}

\section{Contraste estadístico de la hipótesis general}

\section{Hipótesis de investigación}

"Existen relación directa y significativa entre la gestión organizacional de atención a la diversidad y la convivencia escolar en las instituciones educativas públicas de nivel secundaria de la Selva Central".

$\mathrm{Al}$ ser muestra grande $(\mathrm{n}>30)$ y las variables ordinales, la contrastación estadística de la hipótesis se realiza con la prueba Z de Gauss para la significancia del coeficiente de correlación por rangos rho de Spearman, cuya estadística de prueba es:

$$
Z=r \sqrt{n-1}
$$

donde $r$ es el coeficiente de correlación por rangos rho de Spearman entre la gestión organizacional y la convivencia escolar calculado con:

$$
r=1-\frac{6 \sum_{i=1}^{n} d_{i}^{2}}{n^{3}-n}, r=1-\frac{6 S}{n^{3}-n}, S=\sum_{i=1}^{n} d_{i}^{2}
$$

El programa SPSS V.26 reporta los resultados de la Tabla 10, donde el coeficiente de correlación por rangos rho de Spearman muestral entre las variables es 0.793 con valor $\mathrm{p}$ de 0 . 
Tabla 10. Evaluación de la significación estadística del coeficiente por rangos rho de Spearman entre gestión organizacional y convivencia escolar.

\begin{tabular}{llcc}
\hline \multicolumn{1}{c}{ Variables } & \multicolumn{1}{c}{ Estadísticos } & Gestión organizacional & Convivencia escolar \\
\hline Gestión organizacional & Coeficiente de correlación & 1 & $0,793^{* *}$ \\
& Sig. (unilateral) & & 0 \\
Convivencia escolar & $\mathrm{n}$ & 480 & 480 \\
& Coeficiente de correlación & $0,793^{* *}$ & \\
& Sig. (unilateral) & 0 & 480 \\
\hline
\end{tabular}

${ }^{* \star}$ La correlación es significativa en el nivel 0,01 (unilateral).

Con estos resultados se rechaza la hipótesis nula $\mathrm{H}_{0}$ y se acepta la hipótesis alternativa $\mathrm{H}_{1}$, descritas como:

$\mathrm{H}_{1}$ : Existe relación directa y significativa entre la gestión organizacional de atención a la diversidad y la convivencia escolar en las instituciones educativas públicas de nivel secundaria de la Selva Central $\left(\mathrm{H}_{1}: \rho>0\right)$.

$\mathrm{H}_{0}$ : No existerelación entrela gestión organizacional de atención a la diversidad y la convivencia escolar en las instituciones educativas públicas de nivel secundaria de la Selva Central $\left(\mathrm{H}_{0}\right.$ : $\rho=0)$.

Aquí, $\rho$ es el coeficiente de correlación por rangos rho de Spearman poblacional entre la gestión organizacional de atención a la diversidad y la convivencia escolar en las instituciones educativas públicas de nivel secundaria de la Selva Central. Con estos resultados se acepta la hipótesis general de investigación.

\section{Contraste estadístico de la primera hipótesis específica}

\section{Hipótesis de investigación}

"Existe relación directa y significativa entre la gestión organizacional de atención a la diversidad y la convivencia escolar, por UGEL y tamaño de institución educativa, en las instituciones educativas públicas de nivel secundaria de la Selva Central."

En vista de que la muestra es grande $(n>30)$ y las variables son ordinales, el contraste estadístico de la hipótesis se realiza con la prueba $\mathrm{Z}$ de Gauss para la significancia del coeficiente de correlación por rangos rho de Spearman, cuya función pivotal es:

$$
Z=r \sqrt{n-1}
$$

Donde $\mathrm{r}$ es el coeficiente de correlación por rangos rho de Spearman entre la gestión organizacional y la convivencia escolar obtenido con: 
$r=1-\frac{6 \sum_{i=1}^{n} d_{i}^{2}}{n^{3}-n}, r=1-\frac{6 S}{n^{3}-n}, S=\sum_{i=1}^{n} d_{i}^{2}$

Las correlaciones entre la gestión organizacional

de atención frente a la diversidad y la convivencia escolar por UGEL y tamaño de institución educativa con la evaluación de su significancia estadística se detallan en la Tabla 11.

Tabla 11. Coeficientes de correlación por rangos rho de Spearman entre la gestión organizacional de atención frente a la diversidad y la convivencia escolar, por UGEL y tamaño de institución educativa $(\mathrm{n}=480)$.

\begin{tabular}{llccc}
\hline \multicolumn{1}{c}{ Criterios } & \multicolumn{1}{c}{ Categoría } & $\mathbf{n}$ & Coeficiente rho de Spearman & Sig. (unilateral) (valor p) \\
\hline UGEL & Chanchamayo & 290 & $0,801^{* *}$ & 0 \\
& Pangoa & 69 & $0,800^{* *}$ & 0 \\
& Pichanaki & 10 & $0,794^{* *}$ & 0 \\
& Río Ene Mantaro & 11 & $0,741^{* *}$ & 0 \\
& Río Tambo & 24 & $0,832^{* *}$ & 0 \\
& Satipo & 76 & $0,737^{* *}$ & 0 \\
Tamaño de I.E. & Grande & 112 & $0,795^{* *}$ & 0 \\
& Mediana & 221 & $0,742^{* *}$ & 0 \\
& Pequeña & 147 & $0,808^{* *}$ & 0 \\
\hline
\end{tabular}

${ }^{(*)}$ Significativa al $1 \%(\mathrm{p}<0.01)$.

Los coeficientes de correlación son todos positivos y altamente significativos, entre 0,737 (Satipo) y 0,832 (Río Tambo).

Con estos resultados se acepta la primera hipótesis específica de investigación.

\section{Contraste estadístico de la segunda hipótesis específica}

\section{Hipótesis de investigación}

"Existen diferencias significativas en el nivel de la gestión organizacional de atención a la diversidad en las instituciones educativas públicas de nivel secundaria de la Selva Central por UGEL y tamaño de la institución educativa”.
Con esta hipótesis se desea comparar el nivel de la gestión organizacional de atención a la diversidad en las instituciones educativas públicas de nivel secundaria de la Selva Central por UGEL y tamaño de la institución educativa, por lo que se recurre a la prueba Chi cuadrado de Pearson para la homogeneidad de muestras grandes ( $\mathrm{n}>30$ ), cuya estadística de prueba es:

$$
\chi^{2}=\sum_{i=1}^{F} \sum_{j=1}^{C} \frac{\left(O_{i j}-E_{i j}\right)^{2}}{E_{i j}}
$$

Esta función tiene distribución Chi Cuadrada con $(\mathrm{F}-1)^{\star}(\mathrm{C}-1)$ grados de libertad, donde $\mathrm{F}$ es el número de filas y $\mathrm{C}$ es el número de columnas de 
la tabla de contingencia de $\mathrm{F}$ filas y $\mathrm{C}$ columnas que resumen las variables nivel de la gestión organizacional (deficiente, aceptable y buena) y UGEL (Chanchamayo, Pangoa, Pichanaki, Río Ene Mantaro, Río Tambo y Satipo) y tamaño de la institución educativa (grande, mediana y pequeña).

La hipótesis nula $\mathrm{H}_{0}$ e hipótesis alternativa $\mathrm{H}_{1}$ a probar son:

$\mathrm{H}_{0}$ "No existen diferencias en el nivel de la gestión organizacional de atención a la diversidad en las instituciones educativas públicas de nivel secundaria de la Selva Central por UGEL y tamaño de la institución educativa”.

$\mathrm{H}_{1}$ : "Existen diferencias significativas en el nivel de la gestión organizacional de atención a la diversidad en las instituciones educativas públicas de nivel secundaria de la Selva Central por UGEL y tamaño de la institución educativa”.

Como el nivel de la gestión organizacional tiene dos categorías $(\mathrm{F}=2)$, las UGEL son seis $(\mathrm{C}=6)$ y el tamaño de la institución educativa son tres (C
=3), entonces la distribución Chi cuadrado tiene dos, diez y cuatro grados de libertad, para comparar el nivel de la gestión organizacional por cargo laboral, UGEL y tamaño de institución educativa, respectivamente. Con ello, al 95\% de confianza estadística, los valores teóricos respectivos de la Chi Cuadrado son 5.991, 18.31 y 9.488.

En términos de los valores calculado y teórico, se aceptará la hipótesis alternativa $\mathrm{H}_{1}$, en contra de la hipótesis nula $\mathrm{H}_{0}$, si el valor calculado de la Chi Cuadrado es mayor que 5.991, 18.31 o 9.488 para comparar el nivel de la gestión organizacional por UGEL y tamaño de la institución educativa, respectivamente; en caso contrario, $\mathrm{H}_{1}$ será rechazada. En términos del valor p, la hipótesis alternativa $\mathrm{H}_{1}$ será aceptada, en contra de la hipótesis nula $\mathrm{H}_{0}$, si el valor $\mathrm{P}$ es menor que el nivel de significación de 0,05.

La contrastación estadística de la comparación del nivel de la gestión organizacional por UGEL y tamaño de la institución educativa se ilustra en la Tabla 12, donde los valores p son $0.395,0.225$ y 0.019 , los dos primeros mayores y el tercero menor que el nivel de significación de 0.05 .

Tabla 12. Prueba chi cuadrado para el nivel de la gestión organizacional de atención a la diversidad por criterios de comparación.

\begin{tabular}{ccccc}
\hline $\begin{array}{c}\text { Criterio de } \\
\text { comparación }\end{array}$ & Chi cuadrado & Grados de libertad & $\begin{array}{c}\text { Significación } \\
\text { asintótica }\end{array}$ & Conclusión \\
\hline UGEL & 12.982 & 10 & 0.225 & Niveles homogéneos \\
Tamaño de I.E. & 11.760 & 4 & 0.019 & Niveles heterogéneos \\
\hline
\end{tabular}


Con estos resultados se rechaza la hipótesis de la comparación del nivel de la gestión organizacional de atención a la diversidad por UGEL y se acepta la hipótesis de la comparación del nivel de la gestión organizacional por tamaño de institución educativa; es decir, los niveles de la gestión organizacional de atención a la diversidad son homogéneos o iguales por UGEL, y son heterogéneos o diferentes por tamaño de institución educativa, favorables a las instituciones medianas y pequeñas, con lo cual se rechaza la segunda hipótesis específica de investigación.

\section{Contraste estadístico de la tercera hipótesis específica}

\section{Hipótesis de investigación:}

"Existen diferencias significativas en el nivel de la convivencia escolar en las instituciones educativas públicas de nivel secundaria de la Selva Central por UGEL y tamaño de la institución educativa”.

Con esta hipótesis se desea comparar el nivel de la convivencia escolar en las instituciones educativas públicas de nivel secundaria de la Selva Central por UGEL y tamaño de la institución educativa, por lo que se recurre a la prueba chi cuadrado de Pearson para la homogeneidad de muestras grandes ( $\mathrm{n}>$ 30), cuya estadística de prueba es:

$$
\chi^{2}=\sum_{i=1}^{F} \sum_{j=1}^{C} \frac{\left(O_{i j}-E_{i j}\right)^{2}}{E_{i j}}
$$

Esta función tiene distribución chi cuadrada con $(\mathrm{F}-1)^{\star}(\mathrm{C}-1)$ grados de libertad, donde $\mathrm{F}$ es el número de filas $\mathrm{y} \mathrm{C}$ es el número de columnas de la tabla de contingencia de F filas y C columnas que resumen las variables nivel de la convivencia escolar (desfavorable, poco favorable y favorable), y UGEL (Chanchamayo, Pangoa, Pichanaki, Río Ene Mantaro, Río Tambo y Satipo) y tamaño de la institución educativa (grande, mediana y pequeña).

La hipótesis nula $\mathrm{H}_{0}$ e hipótesis alternativa $\mathrm{H}_{1}$ a probar son:

$\mathrm{H}_{0}$ "No existen diferencias en el nivel de la convivencia escolar en las instituciones educativas públicas de nivel secundaria de la Selva Central por UGEL y tamaño de la institución educativa".

$\mathrm{H}_{1}$ : "Existen diferencias significativas en el nivel de la convivencia escolar en las instituciones educativas públicas de nivel secundaria de la Selva Central por UGEL y tamaño de la institución educativa”.

Como el nivel de la convivencia escolar tiene dos categorías $(\mathrm{F}=2)$ las UGEL son seis $(\mathrm{C}=6) \mathrm{y}$ el tamaño de la institución educativa son tres $(\mathrm{C}=$ 3), entonces la distribución chi cuadrado tiene dos, diez y cuatro grados de libertad, para comparar el nivel de la convivencia escolar por UGEL y tamaño de institución educativa, respectivamente. Con ello, al 95\% de confianza estadística, los valores teóricos respectivos de la Chi cuadrado son 5.991, 18.31 y 9.488 .

En términos de los valores calculado y teórico, se aceptará la hipótesis alternativa $\mathrm{H}_{1}$, en contra de la hipótesis nula $\mathrm{H}_{0}$, si el valor calculado de la Chi Cuadrado es mayor que 5.991, 18.31 o 9.488 para comparar el nivel de la gestión organizacional por UGEL y tamaño de la institución educativa, respectivamente; en caso contrario, $\mathrm{H}_{1}$ será rechazada. En términos del valor p, la hipótesis alternativa $\mathrm{H}_{1}$ será aceptada, en contra de la hipótesis nula $\mathrm{H}_{0}$, si el valor $\mathrm{P}$ es menor que el nivel de significación de 0,05. 
La contrastación estadística de la comparación del nivel de la convivencia escolar por UGEL y tamaño de la institución educativa se ilustra en la
Tabla 13, donde los valores $\mathrm{p}$ son $0.436,0.736 \mathrm{y}$ 0.036 , los dos primeros mayores y el tercero menor que el nivel de significación de 0.05 .

Tabla 13. Prueba Chi cuadrado para el nivel de la convivencia escolar por criterios de comparación.

\begin{tabular}{ccccc}
\hline $\begin{array}{c}\text { Criterios de } \\
\text { comparación }\end{array}$ & Chi cuadrado & Grados de libertad & $\begin{array}{c}\text { Significación } \\
\text { asintótica }\end{array}$ & Conclusión \\
\hline UGEL & 6.886 & 10 & 0.736 & Niveles homogéneos \\
Tamaño de I.E. & 10.298 & 4 & 0.036 & Niveles heterogéneos \\
\hline
\end{tabular}

Con estos resultados se rechaza la hipótesis de la comparación del nivel de la convivencia escolar por UGEL y se acepta la hipótesis de la comparación del nivel de la convivencia escolar por tamaño de institución educativa; es decir, los niveles de la convivencia escolar de atención a la diversidad son homogéneos o iguales por UGEL, y son heterogéneos o diferentes por tamaño de institución educativa, favorables a las instituciones medianas y pequeñas, con lo cual se rechaza la tercera hipótesis específica de investigación.

El análisis correlacional demostró una relación directa, fuerte $(0,793)$ y altamente significativa $(* *$, $\mathrm{p}<0,01)$ entre la gestión organizacional de atención a la diversidad y la convivencia escolar; lo que implica, a mejor gestión organizacional de atención a la diversidad, mejor es la convivencia escolar en la institución educativa.

Por su parte, el análisis correlacional por UGEL demostró la relación entre la gestión organizacional y la convivencia escolar es directa, fuerte y altamente significativa, entre 0,737 (Satipo) y 0,832 (Río Tambo), y por tamaño de institución educativa, la relación entre la gestión organizacional y la convivencia escolar es directa, fuerte y altamente significativa, entre 0,742 en instituciones medianas y 0,808 en instituciones educativas pequeñas asentadas en la Selva Central.
La gran mayoría de los colaboradores perciben un nivel bueno de gestión organizacional de atención a la diversidad, y un nivel favorable de convivencia escolar en las instituciones educativas públicas de nivel secundaria de la Selva Central.

El nivel de la gestión organizacional de atención a la diversidad percibida por tamaño de institución educativa es buena y ligeramente mayor en las instituciones medianas, que en las instituciones pequeñas y grandes, y el nivel de la gestión organizacional de atención a la diversidad percibida por UGEL es buena y ligeramente mayor en UGEL Pangoa (94,2\%) y UGEL Río Tambo (91,7\%), que en UGEL Chanchamayo (84,5\%), UGEL Pichanaki (80\%), UGEL Satipo (80,3\%) y UGEL Río Ene Mantaro (63,6\%).

El nivel de la convivencia escolar en las instituciones educativas percibida por tamaño de institución educativa es favorable y ligeramente mayor en las instituciones pequeñas, que en las instituciones medianas y las grandes, y el nivel de la convivencia escolar percibida por UGEL es favorable y superior al $90 \%$ en todas Unidades de Gestión Educativa Local asentadas en la Selva Central. 


\section{Discusión}

Abordando el análisis de los resultados estadísticos del estudio, con la investigación científica precedente y las bases teóricas sustentadas; persiguiendo el propósito de determinar la relación existente entre la gestión organizacional de atención a la diversidad y la convivencia escolar. Los primeros resultados al contrastar las hipótesis con el coeficiente correlación por rangos rho de Spearman, se hallaron una relación directa y fuerte (0.793) y altamente significativa $\left(^{* *}, \mathrm{p}<0,01\right)$ entre las variables de estudio; lo que implica, a mejor gestión organizacional de atención a la diversidad, mejor es la convivencia escolar. Estos resultados parecen coincidir con la investigación realizada por Ramírez (2017) que refiere la gestión institucional se relaciona positiva y significativamente con la convivencia escolar; lo que implica, el gobierno escolar inherente a la gestión institucional genera una convivencia escolar favorable.

Al concertar los resultados con la investigación realizada por Vargas (2019) demostró una correlación altamente significativa entre la gestión de la diversidad y la calidad del servicio educativo, lo que implica a mejor gestión de la diversidad, mayores son las condiciones básicas de atención; asimismo los estudios de Bustamante (2019) demostraron que la gestión escolar, el liderazgo directivo guarda relación significativa con la convivencia escolar; esto significa, los mecanismos de comunicación, las alianzas interinstitucionales, el gobierno escolar inherente a la gestión institucional favorecen una convivencia escolar armoniosa.

Por otro lado, los resultados estadísticos de esta investigación reflejan un nivel bueno (85\%) de gestión organizacional de atención a la diversidad en las instituciones educativas de nivel secundaria de la Selva Central. Asimismo, el nivel de la convivencia escolar percibido por los colaboradores es favorable $(94,4 \%)$. Estos hallazgos encuentran respaldo en los estudios de Arias (2018) y Quispe et al. (2018) al señalar que el respeto por la diversidad, las relaciones horizontales, el compromiso y mediación de los actores educativos, constituyen estrategias de fortalecimiento de la convivencia escolar favorable. Asimismo, tiene sustento en los estudios de Quispe, Robinet y López (2018) al demostrar que la aplicación de las normas, la participación colectiva, las decisiones consensuadas, la inclusión y el respeto a la diversidad en la práctica de la convivencia escolar; así como el trato horizontal entre estudiantes y profesores propician una convivencia democrática e inclusiva favorable al interior de la institución educativa. No obstante, las investigaciones de Bustamante (2019) parecen ser contradictorios con los estudios realizados al referirse que la menoría (30\%) de los docentes perciben la convivencia escolar en un nivel buena, como consecuencia de la gestión educativa de un nivel moderadamente eficiente.

En lo que respecta al nivel de la gestión organizacional de atención a la diversidad en las instituciones educativas, por tamaño de institución educativa es buena y ligeramente mayor en las instituciones medianas $(88,7 \%)$, que en las instituciones pequeñas $(85,7 \%)$ y grandes $(76,8 \%)$, y el nivel de la gestión organizacional de atención a la diversidad por UGEL es buena y ligeramente mayor en UGEL Pangoa (94,2\%) y UGEL Río Tambo (91,7\%), que en UGEL Chanchamayo (84,5\%), UGEL Pichanaki (80\%), UGEL Satipo (80,3\%) y UGEL Río Ene Mantaro (63,6\%). Estos resultados estarían reflejando los efectos del Programa Nacional de Formación y Capacitación de Directores y Subdirectores de Instituciones Educativas; así como del Programa de formación continua para docentes de educación básica en servicio que han recibido los directivos y docentes 
de parte del Ministerio de Educación; asimismo, reflejan los efectos de la aplicación de las normativas educativas nacionales, regionales y locales, $y$ principalmente de la planificación y aplicación de los instrumentos de gestión institucional (Proyecto educativo institucional, plan anual de trabajo y reglamento interno), así también de la adecuación y adaptación curricular en el aula, y sin duda, del compromiso e involucramiento del equipo directivo y docente.

Estos resultados son concordantes con los estudios de Luzón et al (2009) al corroborar que los profesores desarrollan "buenas prácticas pedagógicas" para dar respuesta a los riesgos de exclusión escolar en las instituciones de nivel secundaria; lo que implica, la presencia del equipo docente altamente motivado y comprometido, con espíritu innovador y profesionalismo para atender la diversidad; así como el equipo directivo con liderazgo educativo y formación pedagógica, que impulsan la marcha de proyectos integrados para generar un buen nivel de organización de atención a la diversidad. Sin embargo, contradiciendo a los resultados de la investigación, Begue (2018) ha encontrado limitaciones en los maestros de la Comunidad de Madrid para poder llevarla a la práctica la inclusión de los estudiantes en las aulas; además en la percepción de los padres de familia, dichos profesores muestran actitudes negativas frente a la diversidad, lo que dificultan la inserción de sus hijos en la escuela ordinaria. Del mismo modo, los estudios realizados en Chile por Jiménez y Montecinos (2018) develaron insuficiente formación inicial del maestro en competencias pedagógicas para laborar en contextos educativos diversos. Bajo esta perspectiva, el rol del docente y su formación inicial y en servicio, cobra una singular relevancia en las prácticas pedagógicas para dar respuesta a la diversidad en el aspecto organizativo, metodológico y curricular (Habib y Hernández, 2020).

Por otro lado, los resultados estadísticos, respecto al nivel de la convivencia escolar en las instituciones educativas, por tamaño de la institución educativa es favorable y ligeramente mayor en las instituciones pequeñas $(96,6 \%)$ que en las instituciones medianas $(95,9 \%)$ y las grandes $(88,4 \%)$, y el nivel de convivencia por UGEL es favorable, ligeramente superior en UGEL Pichanaki y Rio Tambo (100\%) que en UGEL Pangoa $(98,6)$, UGEL Chanchamayo (93,4\%), UGEL Satipo (92,1\%) y UGEL Río Ene Mantaro (90,9). Estos resultados estarían reflejando los efectos del Programa Nacional de Formación y Capacitación de Directores y Subdirectores de Instituciones Educativas; así como del Programa de formación continua para docentes de educación básica en servicio que han recibido los directivos y docentes de parte del Ministerio de Educación; además de los efectos de la aplicación de las normativas educativas nacionales, regionales y locales, y principalmente de la planificación y aplicación de los instrumentos de gestión institucional (Proyecto educativo institucional, plan anual de trabajo y reglamento interno), así como de la construcción y aplicación de las normas de convivencia en las instituciones educativas, $\mathrm{y}$ sin duda del compromiso e involucramiento del equipo directivo y docente.

Estos hallazgos parecen coincidir con las investigaciones de Arias (2018) al revelar que los ambientes escolares planificados y adecuados, establecen relaciones favorables que fortalecen la convivencia escolar. No obstante, parecen ser contradictorios a nuestra investigación, los hallazgos de Aragón (2019) al referirse a los 
profesores que no están capacitados para abordar los problemas de convivencia que se manifiestan en el contexto escolar; Así como, los estudios realizados en Chile por Retuert y Castro (2017) y MoncadaHerrera (2018) revelan la necesidad de fortalecer la formación inicial y en servicio de los profesores para nutrir las competencias didácticas que ayude a regular la disciplina en el aula y fomentar ambientes armoniosos de convivencia escolar.

\section{CONCLUSIONES}

Con base a los resultados del estudio, se recomienda proponer un plan de acción para el fortalecimiento de la gestión organizacional de atención a la diversidad y gestión de la convivencia escolar en las instituciones educativas de educación básica. Asimismo, proponer al Ministerio de Educación y a los órganos descentralizados, impulsar la formación continua del magisterio para fortalecer sus competencias pedagógicas y de gestión en la atención a la diversidad y convivencia escolar.

\section{REFERENCIAS}

Álvarez, Q. (2018). Pedagogía sistémica e interculturalidad: Claves para construir un aula inclusiva. Revista Lusófona de Educação, 37,165179

Aragón, A. (2019). La convivencia escolar: una mirada desde la educación social. Tesis de grado. Universidad de Valladolid

Arias, I. C. (2018). Ambientes escolares: un espacio para el reconocimiento y respeto por la diversidad. Sophia, 14(2), 84-93. https://doi. org/10.18634/sophiaj.14v.2i.852

Arnaiz, P. (2003). Multiculturalidad y diversidad en las aulas. Indivisa. Boletín de Estudios e Investigación, 4, 9-30

Begué, A. P. (2018). La atención a la diversidad en los centros educativos de la Comunidad de Madrid. Análisisde de la relación entre el texto $y$ el contexto. (Tesis doctoral, Universidad Complutense de Madrid). https://eprints.ucm. es/id/eprint/49393/1/T40282.pdf

Bustamante, A. (2019). Gestión educativa y convivencia escolar en el colegio "Cesar A. Vallejo" - La Victoria, 2018. (Tesis de maestría, Universidad Cesar Vallejo). https://repositorio. ucv.edu.pe/handle/20.500.12692/31945

Castro-Rodríguez, M. M., y Eirín-Nemiña, R. (2018). Evaluación de materiales de educación física para la atención a la diversidad. Análisis de una propuesta. Retos, 2041 (34), 356-362. https://doi.org/10.47197/retos.v0i34.65894

Cepeda, J. (2018). Pensando el enigma de la subjetividad a través de la diversidad. Sophía, 25(25), 233-258. https://doi.org/10.17163/soph. n25.2018.08

Congreso de la República (2003). Ley General de Educación. Ley $N^{\circ}$ 28044. Recuperado de http://www.minedu.gob.pe/p/ley_general_de_ educacion_28044.pdf

Consejo Nacional de Educación (2020). Proyecto educativo nacional al 2036. Ministerio de Educación. https://bit.ly/2WOS9wR

Cornejo, C. (2017). Respuesta educativa en la atención a la diversidad desde la perspectiva de profesionales de apoyo. Revista Colombiana de Educación, (73), 75-94

Cornejo Valderrama, C., Lepe Martínez, N., y Vidal Espinosa, R. (2017). Respuesta educativa en la atención a la diversidad desde la perspectiva de profesionales de apoyo. Revista Colombiana de Educación, (73), 75. https://doi.org/10.17227/01 203916.73rce75.94

Fregoso, M. V. (2019). Aspectos interculturales en los libros de texto gratuitos de primaria, en México. 405-422

Habib, M. C., y Hernández, A. (2020). Actitudes de los escolares ante la llegada de población migrante: Estudio comparativo y aproximación al desarrollo de buenas prácticas educativas. Educatio Siglo XXI, 38, 229-252. https://doi. org/10.6018/educatio.413491 
Hernández Prados, M. Á., e Ibáñez Bordallo, D. (2018). La formación del profesorado ante la diversidad de creencias religiosas en el máster de formación de profesorado de enseñanza secundaria. Percepción docente. Cuadernos de Investigación Educativa, 9(1), 59. https://doi. org/10.18861/cied.2018.9.1.2821

Jiménez, F., y Montecinos, C. (2018). Diversidad, modelos de gestión y formación inicial docente: desafíos formativos desde una perspectiva de justicia social. Revista Brasileira de Educação, 23(0), 1-21. https://doi.org/10.1590/s141324782018230005

Luzón, A., Porto, M., Torres, M., y Ritacco, M. (2009). Buenas prácticas en los programas extraordinarios de atención a la diversidad en centros de educación secundaria. Una mirada desde la experiencia. Revista de currículum y formación del profesorado, 13(3), 217-238

Magro-Gutiérrez, M. (2019). Necesidades formativas del profesorado de educación infantil para la atención a la diversidad de colegios rurales agrupados. Revista Prisma Social, 25,103-125

Maldonado, E. P. (2018). Competencias del profesorado universitario para la atención a la diversidad en la educación superior. Revista Latinoamericana de Educación Inclusiva, 12(2), 115-131. https://doi.org/10.4067/s071873782018000200115

Ministerio de Educación (2020). Resolución Viceministerial $\quad N^{\circ}$ 273-2020-MINEDU. Orientaciones para el desarrollo del Año Escolar 2021 en Instituciones Educativas y Programas Educativos de la Educación Básica.

Moncada-Herrera, J. (2018). Gestión convivencia escolar: del aseguramiento a la necesidad de fortalecer la formación socioemocional. https:// bit.ly/3h7Y1YP

Morin, E. (2000). Los siete saberes necesarios para la educación del futuro. Barcelona: Paidós. https:// bit.ly/3jLA779
Ochoa, A., y Perez, L. (2019). El aprendizaje servicio, una estrategia para impulsar la participación y mejorar la convivencia escolar. Psicoperspectivas, 18(1), 1-13. https://doi. org/10.5027/psicoperspectivas-vol18-issue1fulltext-1478

Organización de las Naciones Unidas. (2017). Objetivos de desarrollo sostenible y politicas de Estado del acuerdo nacional. https://bit. ly/3BGASVe

Organización de las Naciones Unidas (1959). Declaración de los derechos del niño, 1959. New York, NY: Autor. https://bit.ly/38KyfVS

Organización de las Naciones Unidas (1999). Declaración y el programa de acción sobre una cultura de paz (53a Asamblea General). New York, NY: Autor. Recuperado de http://www.undocuments.net/a53r243.htm

Quispe, M. N., Robinet, S. M., y Lopez, S. (2018). Significados y práctica de una convivencia escolar en una IEP de Ayacucho. (Tesis de maestría, Pontificia Universidad Católica del Perú). https://bit.ly/3BLh6rO

Ramírez, J. (2017). Gestión institucional y convivencia escolar en la Institución Educativa "Alberto Leveaú García" de la provincia de Picota - 2016. (Tesis de maestría, Universidad César Vallejo). https://bit.ly/3n6pwWK

Retuert, G., y Castro, P. J. (2017). Teorías subjetivas de profesores acerca de su rol en la construcción de la convivencia escolar. Polis (Santiago), 16(46), 321-345. https://doi.org/10.4067/s071865682017000100321

Rojas, V. (2020). Habilidades blandas en currículo educativo. ( $1^{a}$ ed.). Perú. https://bit.ly/3h4JX21

Vargas, J. (2019). La gestión de la diversidad y la calidad del servicio educativo en los colegios de la provincia de Lucanas, región Ayacucho. (Tesis Doctoral, Universidad Nacional de Educación «Enrique Guzmán y Valle"). https:// bit.ly/3jLEhfl 